\title{
ON SEMI-CYLINDERS, SPLINTERS, AND BOUNDED-TRUTH-TABLE REDUCIBILITY
}

\author{
BY \\ PAUL R. YOUNG $\left({ }^{1}\right)$
}

In Part I of this paper we construct a bounded-truth-table-complete set which is not creative, and in Part II we construct an infinite and coinfinite recursively enumerable (r.e.) splinter which is not a cylinder. Although these results appear unrelated, the proofs given here are similar and so have been included in a single paper. The two parts may be read independently of one another. Each of these results yields as corollary the existence of pseudo-creative sets which are not cylinders. In fact, in Part II we construct a pseudo-creative set $S$ for which there is no total recursive function $f$ such that $x \in S$ implies $f(x) \in S-\{x\}$ and $x \in S^{\prime}$ implies $f(x) \in S^{\prime}-\{x\}$.

Preliminaries. We will use $N$ to denote the set of all non-negative integers. Unless specifically mentioned otherwise, all sets are considered subsets of $N$. If $A$ is a set, $A^{\prime}=N-A$. Since we consider only sets of integers, we will not use Cartesian products of sets but will instead work with images of Cartesian products under some effective mapping. More specifically, if $A$ and $B$ are subsets of $N$, let $A \otimes B=\{(a, b) \mid a \in A$ and $b \in B\}$. Let $\tau$ be any effective one-to-one mapping of $N \otimes N$ onto $N$. Then we define $A \times B$ to be $\tau(A \otimes B)$, and we abbreviate $\tau((a, b))$ to $\langle a, b\rangle$. (This is the notation introduced by Rogers in [5].) Given integers $a$ and $b$ we can always effectively find the integer $\langle a, b\rangle$, and given the integer $\langle a, b\rangle$ we can always effectively find $a$ and $b$.

$\phi_{e}$ is the partial recursive function with index $e$. If $\phi_{e}$ is defined for every member of $N$, we say that $\phi_{e}$ is total.

In [3], Myhill has called a set a cylinder if it is recursively isomorphic to $B \times N$ for some r.e. set $B$, however we will follow Rogers in calling a set a cylinder if it is recursively isomorphic to $B \times N$ for any set $B$. Let $D_{x}$ be the nonempty finite set with canonical index $x$. In [5], Rogers shows that a set $A$ is a cylinder if and only if there is a total recursive function $f$ such that $D_{x} \subset A$ implies $f(x) \in A-D_{x}$ and $D_{x} \subset A^{\prime}$ implies $f(x)$ $\in A^{\prime}-D_{x}$.

Part I was presented to the Society, August 29, 1963, under the title A bounded-truth-tablecomplete, pseudo-creative, noncylinder, and Part II, January 23, 1964, under the title $A$ recursively enumerable splinter which is not a cylinder; received by the editors December 13, 1963.

( ${ }^{1}$ ) The research reported in Part I of this paper forms a portion of the author's doctoral dissertation, written at the Massachusetts Institute of Technology under the direction of Professor Hartley Rogers, Jr., and was supported by a National Science Foundation Fellowship. 
Definition. A set $A$ is a semi-cylinder if there is a total recursive function $h$ such that $x \in A$ implies $h(x) \in A-\{x\}$ and $x \in A^{\prime}$ implies $h(x)$ $\in A^{\prime}-\{x\}$. If the function $h$ can be taken one-to-one, we say that $A$ is a 1-1 semi-cylinder.

It is readily shown that if $A$ is a recursive set, $A$ is a semi-cylinder if and only if neither $A$ nor $A^{\prime}$ is a unit set.

Lemma. Every cylinder is a 1-1 semi-cylinder. Every 1-1 semi-cylinder is a cylinder.

Proof. The second statement is obvious. To prove the first, let $A$ be a cylinder. We may assume $A=B \times N$ for some set $B$. Define $h(\langle a, n\rangle)$ $=\langle a, n+1\rangle$ for all $a$ and $n$. $h$ is then the required function.

We can also show that 1-1 semi-cylinders and cylinders differ on the r.e. sets: If $S$ is an infinite r.e. set, $S \times S$ is recursively isomorphic to $S \times S$ $\cup\{\langle n, n\rangle \mid n \in N\} .(S \times S) \cup\{\langle n, n\rangle \mid n \in N\}$ is easily shown to be a 1-1 semi-cylinder, and in [7] we have shown the existence of a simple set, $S_{0}$, for which $S_{0} \times S_{0}$ is not a cylinder.

Lemma (Myнill). Every creative set is a cylinder.

Proof. Suppose $K$ is creative. Since every r.e. set is 1-1 reducible to every creative set, $K \times N$ is $1-1$ reducible to $K$. For any set $A, A$ is 1-1 reducible to $A \times N$; thus $K$ is $1-1$ reducible to $K \times N$, and so $K$ is recursively isomorphic to $K \times N$.

\section{A BOUNDED-TRUTH-TABLE-COMPLETE SET WHICH IS NOT CREATIVE}

Introduction. Let $R$ be any of the reducibility relations introduced by Post in [4]. Post called a r.e. set $R$-complete if every r.e. set is $R$-reducible to it, and he proved that the class of Turing-complete sets, the class of truth-table-complete sets, and the class of bounded-truth-table-complete sets are all distinct. In [2], Myhill shows that the class of many-onecomplete sets and the class of one-one-complete sets are each identical with the class of all creative sets.

Recently Fischer has shown that bounded-truth-table reducibility differs from many-one reducibility on the r.e. sets [1], but the question of whether every bounded-truth-table-complete set is creative (i.e., of whether the class of bounded-truth-table-complete sets is identical with the class of many-one-complete sets) has remained open.

In Part I we construct a bounded-truth-table-complete set which is not creative and obtain as corollaries a new proof of Fischer's result and the existence of a pseudo-creative set which is not a cylinder.

Lemma. If $A$ is a cylinder, then there exists a recursive permutation $t$ such that

(i) for all $x, t(x) \neq x$,

(ii) for all $x, t^{2}(x)=x$, and 
(iii) for all $x, x \in A$ if and only if $t(x) \in A$.

Proof. We may assume $A=B \times N$ for some set $B$. Define $t(\langle y, 2 n\rangle)$ $=\langle y, 2 n+1\rangle$ and $t(\langle y, 2 n+1\rangle)=\langle y, 2 n\rangle$.

THEOREM. Any creative set, $K$, is the disjoint union of two recursively isomorphic, r.e., sets, $L_{0}$ and $L_{1}$, which are not 1-1 semi-cylinders.

Discussion. Since all creative sets are recursively isomorphic, we may assume that $K=\left\{x \mid \phi_{x}(x)\right.$ is defined $\}$. Since all creative sets are cylinders, there exists a 1-1 recursive permutation $t$ such that

(i) for all $x, t(x) \neq x$,

(ii) for all $x, t^{2}(x)=x$, and

(iii) for all $x, x \in K$ if and only if $t(x) \in K$.

Let $h$ be a 1-1 recursive function whose range is $K$. Define $g(2 n)=h(y)$, where $y$ is the least $z$ such that

$$
h(z) \notin\{g(0), g(1), \cdots, g(2 n-1)\},
$$

and $g(2 n+1)=t(g(2 n))$.

Since $h(z) \in\{g(0), g(1), \cdots, g(2 n-1)\}$ if and only if

$$
t(h(z)) \in\{g(0), g(1), \cdots g(2 n-1)\},
$$

$g$ is a 1-1 recursive function whose range is $K$.

In proving the theorem, we will proceed in stages, constructing the sets $L_{0}$ and $L_{1}$ in such a way that the permutation $t$ will be the required isomorphism between $L_{0}$ and $L_{1}$. A priority argument will be used to assure that $L_{0}$ has no function of the kind described in the definition of 1-1 semicylinders. The idea is to spoil each total recursive function $f$ by finding some index $r$ such that $\phi_{r}=f$ and either

(i) $\phi_{r}(r)=r$,

(ii) $\phi_{r}$ is not a 1-1 function,

(iii) $r \in L_{1}$ and $\phi_{r}(r) \in L_{0}$, or

(iv) $r \in L_{0}$ and $\phi_{r}(r) \notin L_{0}$.

It is easily seen that any one of these conditions is sufficient to assure that $f$ will not be the function required by the definition if $L_{0}$ is to be a 1-1 semi-cylinder.

In constructing the sets $L_{0}$ and $L_{1}$, we imagine the non-negative integers to occur in a list, and as the construction proceeds we associate certain markers with some of the integers in the list. We will use two infinite collections of markers: $\left\{\Lambda_{i}\right\}$ and $\left\{\Lambda_{i}^{\prime}\right\}, 0 \leqq i<\infty$.

At any stage in the construction, for any $i$, at most one of the markers $\Lambda_{i}$ and $\Lambda_{i}^{\prime}$ will be associated with some number. At any stage, any marker is beside at most one number and no number has more than one marker beside it. At Stage $n$, only markers $\Lambda_{i}$ and $\Lambda_{i}^{\prime}$ for $i \leqq 2 n+1$ can be in use. If at the end of Stage $n$, neither $\Lambda_{i}$ nor $\Lambda_{i}^{\prime}$ for $i \leqq 2 n+1$ is in use, then the function $\phi_{g(i)}$ has been spoiled. 
Each stage of the construction will have two steps, Step 1 and Step 2. The first of these will be used to assure that exactly one of $g(2 n)$ and $g(2 n+1)(=t(g(2 n)))$ has a marker beside it. The second step will be used to assure that $L_{0}$ is not a $1-1$ semi-cylinder. The action taken at the second step will depend on the marker which is associated with either $g(2 n)$ or $g(2 n+1)$ at the end of the first step.

Construction and proof. $L_{i}(a)$ will be the set of integers placed in $L_{i}$ by the beginning of Stage $a(i=0,1)$. We now give the construction:

Stage $n(n \geqq 0)$.

Step 1. Compute $g(2 n)$ and $g(2 n+1)(=t(g(2 n)))$.

If neither $g(2 n)$ nor $g(2 n+1)$ has a marker beside it, place the marker $\Lambda_{2 n}$ beside $g(2 n)$. Then find an $r$ such that $\phi_{r}=\phi_{g(2 n+1)}$ and (i) neither $r$ nor $t(r)$ has a marker beside it, and (ii)

$$
r \notin L_{0}(n) \cup L_{1}(n) \cup\{g(2 n), g(2 n+1)\} .
$$

Place $\Lambda_{2 n+1}$ beside $r$ and go on to Step 2 .

If either $g(2 n)$ or $g(2 n+1)$ has a marker beside it, find $r_{0}$ and $r_{1}$ such that $\phi_{r_{0}}=\phi_{g(2 n)}$ and $\phi_{r_{1}}=\phi_{g(2 n+1)}\left(r_{0} \neq r_{1}\right)$ and (i) $t\left(r_{0}\right) \neq r_{1}$ and neither $r_{0}, r_{1}$, $t\left(r_{0}\right)$, nor $t\left(r_{1}\right)$ has a marker beside it, and (ii) neither $r_{0}$ nor $r_{1}$ belongs to $L_{0} \cup L_{1} \cup\{g(2 n), g(2 n+1)\}$. Place the marker $\Lambda_{2 n}$ beside $r_{0}$ and $\Lambda_{2 n+1}$ beside $r_{1}$ and go on to Step 2.

Step 2. After completing Step 1, we find that exactly one of $g(2 n)$ and $g(2 n+1)$ has a marker beside it. We shall assume the marker is beside $g(2 n)$. The case where it is beside $g(2 n+1)$ is treated similarly.

Case A. The marker beside $g(2 n)$ is $\Lambda_{i}$. (So $\phi_{g(2 n)}=\phi_{g(i)}$.) In this case we first compute $\phi_{g(2 n)}(g(2 n))$.

Subcase I. The marker $\Lambda_{i}$ has at some stage in the construction been beside a number $m \neq g(2 n)$, and $\phi_{m}(m)$ was computed and $\phi_{m}(m)=\phi_{g(2 n)}(g(2 n))$.

In this case $\phi_{g(i)}$ is not a 1-1 function which, according to (ii) of our preliminary discussion, spoils $\phi_{g(i)}$. Consequently we erase the marker $\Lambda_{i}$ and place $g(2 n)$ in $L_{0}$ and $g(2 n+1)$ in $L_{1}$ and go on to Stage $n+1$.

Subcase II. $\phi_{g(2 n)}(g(2 n))=g(2 n)$ or $g(2 n+1)$.

In the first of these cases, applying (i) of our preliminary discussion, $\phi_{g(i)}$ is automatically spoiled, so place $g(2 n)$ in $L_{0}$ and $g(2 n+1)$ in $L_{1}$. In the second case, applying (iv) of our preliminary discussion, $\phi_{g(i)}$ can be spoiled by placing $g(2 n)$ in $L_{0}$ and $g(2 n+1)$ in $L_{1}$. In either case, erase $\Lambda_{i}$, and go on to Stage $n+1$.

Subcase III. $\phi_{g(2 n)}(g(2 n)) \in L_{q}(n)(q=0$ or 1$)$.

In this case, applying (iii) and (iv) of our preliminary discussion, spoil $\phi_{g(i)}$ by placing $g(2 n)$ in $L_{1-q}$. Since $\phi_{g(i)}$ is spoiled, erase $\Lambda_{i}$. To preserve the isomorphism $t$ between $L_{0}$ and $L_{1}$, place $g(2 n+1)$ in $L_{q}$, and go on to Stage $n+1$. 
Subcase IV. None of the preceding subcases applies, and neither $\phi_{g(2 n)}(g(2 n))$ nor $t\left(\phi_{g(2 n)}(g(2 n))\right)$ has a marker beside it.

In this case, erase $\Lambda_{i}$ and place $\Lambda_{i}^{\prime}$ beside $\phi_{g(2 n)}(g(2 n))$. Then put $g(2 n)$ in $L_{0}$ and $g(2 n+1)$ in $L_{1}$, and go on to Stage $n+1$.

Subcase V. None of the preceding subcases applies, but either $\phi_{g(2 n)}(g(2 n))$ or $t\left(\phi_{g(2 n)}(g(2 n))\right.$ has beside it a marker $\Lambda_{x}$ or $\Lambda_{x}^{\prime}$ with $x>i$.

In this case find $r$ such that $\phi_{r}=\phi_{g(x)}$, and (i) neither $r$ nor $t(r)$ has a marker beside it, and (ii) $r \notin L_{0}(n) \cup L_{1}(n) \cup\{g(2 n), g(2 n+1)\}$. Erase $\Lambda_{x}$ or $\Lambda_{x}^{\prime}$ and place $\Lambda_{x}$ beside $r$. Then erase $\Lambda_{i}$ and place $\Lambda_{i}^{\prime}$ beside $\phi_{g(2 n)}(g(2 n))$. Finally, put $g(2 n)$ in $L_{0}$ and $g(2 n+1)$ in $L_{1}$, and go on to Stage $n+1$.

Subcase VI. None of the preceding subcases applies. Thus either $\phi_{g(2 n)}(g(2 n))$ or $t\left(\phi_{g(2 n)}(g(2 n))\right)$ has beside it a marker $\Lambda_{x}$ or $\Lambda_{x}^{\prime}$ with $x \leqq i$. (When the description of the construction is completed, it will be clear that $x \neq i$; hence $x<i$.)

In this case, find $r$ such that $\phi_{r}=\phi_{g(i)}$ and (i) neither $r$ nor $t(r)$ has a marker beside it, and (ii) $r \notin L_{0}(n) \cup L_{1}(n) \cup\{g(2 n), g(2 n+1)\}$. Remove $\Lambda_{i}$ from $g(2 n)$ and place it next to $r$. Then put $g(2 n)$ in $L_{1}$ and $g(2 n+1)$ in $L_{0}$ and go on to Stage $n+1$.

Case B. The marker beside $g(2 n)$ is $\Lambda_{i}^{\prime}$. In this case, by examining the construction we see that there exists $r$ such that $\phi_{r}=\phi_{g(i)}, r \in L_{0}$, and $\phi_{r}(r)=g(2 n)$. We spoil $\phi_{g(i)}$ by placing $g(2 n)$ in $L_{1}$. Then we put $g(2 n+1)$ in $L_{0}$, erase the marker $\Lambda_{i}^{\prime}$, and go on to Stage $n+1$.

This completes the description of the construction.

Since $x \in K$ if and only if $x=g(2 n)$ or $g(2 n+1)$ for some $n$, and since $g(2 n) \in L_{i}(i=0$ or 1$)$ if and only if $t(g(2 n))(=g(2 n+1)) \in L_{1-i}$, we easily see that $L_{0} \cap L_{1}=\emptyset, L_{0} \cup L_{1}=K$, and $t\left(L_{0}\right)=L_{1}$ (so $L_{0}$ and $L_{1}$ are recursively isomorphic). Since $L_{0}$ and $L_{1}$ are clearly r.e., we can complete the proof of the theorem by showing that $L_{0}$ is not a 1-1 semi-cylinder.

In the following discussion, when we speak of replacing $\Lambda_{i}$ we mean that $\Lambda_{i}$ is erased and $\Lambda_{i}^{\prime}$ is introduced beside some number. Similarly, when we say that $\Lambda_{i}^{\prime}$ is replaced we mean that $\Lambda_{i}^{\prime}$ is erased and $\Lambda_{i}$ is introduced beside some number. When in the following discussion we say that a marker is erased we mean that it is erased and not replaced.

At Stage 0 , the markers $\Lambda_{0}$ and $\Lambda_{1}$ are introduced. At Step 2 of this stage, the marker $\Lambda_{0}$ is either erased or replaced by $\Lambda_{0}^{\prime}$, but $\Lambda_{0}^{\prime}$, although it can be erased, can never be replaced or moved. This establishes the basis for an inductive argument showing that no marker gets moved infinitely often.

Suppose all markers $\Lambda_{j}$ and $\Lambda_{j}^{\prime}$ with $j<i$ have either been permanently erased or have reached a final resting spot by Stage $n_{0}$. Suppose also that the marker $\Lambda_{i}$ or $\Lambda_{i}^{\prime}$ is moved infinitely often. $\Lambda_{i}^{\prime}$ can be moved only if it is first replaced by $\Lambda_{i}$, so we may suppose that at some Stage $n_{1}\left(n_{1} \geqq n_{0}\right) \Lambda_{i}$ 
is next to some number $r . \Lambda_{i}$ can then be moved or replaced only if $\phi_{r}(r)$ is computed and fails to belong to $L_{0}\left(n_{1}\right) \cup L_{1}\left(n_{1}\right)$ and either

(a) $\phi_{r}(r)$ or $t\left(\phi_{r}(r)\right)$ has beside it a marker $\Lambda_{j}$ or $\Lambda_{j}^{\prime}$ with $j<i$, in which case $\Lambda_{i}$ is moved immediately, or

(b) $\phi_{r}(r)$ and $t\left(\phi_{r}(r)\right)$ have no marker or a marker $\Lambda_{j}$ or $\Lambda_{j}^{\prime}$ with $j>i$ beside them, in which case $\Lambda_{i}^{\prime}$ is placed beside $\phi_{r}(r)$, replacing $\Lambda_{i}$.

Since Case A, Subcase I of Step 2 of the construction is used to spoil functions which are not 1-1, case (a) of the preceding paragraph can occur at most finitely often after Stage $n_{1}$. Consequently, at some Stage $n_{2}$ $\left(n_{2} \geqq n_{0}\right)$, case (b) of the preceding paragraph must occur if $\Lambda_{i}$ or $\Lambda_{i}^{\prime}$ is to be moved infinitely often. But no marker $\Lambda_{j}$ or $\Lambda_{j}^{\prime}$ with $j<i$ ever gets moved after Stage $n_{0}$, so $\Lambda_{i}^{\prime}$ can never be replaced by $\Lambda_{i}$. Thus $\Lambda_{i}^{\prime}$, although it may be erased, can never be replaced by $\Lambda_{i}$. Thus $\Lambda_{i}^{\prime}$ will never appear in another position, and $\Lambda_{i}$ will never appear again. This shows that all markers get moved at most finitely often.

Furthermore, we see that the only way in which $\Lambda_{i}$ can come permanently to rest beside some number $r$ is for $\phi_{r}(r)$ never to be computed. Since $\phi_{r}$ must be $\phi_{g(i)}$, this cannot happen if $\phi_{g(i)}$ is a total function. Thus if $\phi_{g(i)}$ is defined everywhere, $\Lambda_{i}$ cannot come permanently to rest beside some number.

Now if $L_{0}$ were a 1-1 semi-cylinder, there would be a 1-1 total recursive function $\phi_{e}$ such that $x \in L_{0}$ implies $\phi_{e}(x) \in L_{0}-\{x\}$ and $x \in L_{0}^{\prime}$ implies $\phi_{e}(x) \in L_{0}^{\prime}-\{x\}$. If $\phi_{e}$ is defined everywhere, every index for $\phi_{e}$ must belong to $K\left(=\left\{x \mid \phi_{x}(x)\right.\right.$ is defined $\left.\}\right)$. Therefore for some number $n, e=g(2 n)$ or $g(2 n+1)$. Suppose $e=g(2 n)$. (The case where $e=g(2 n+1)$ is treated similarly.) Then at Stage $n$, the marker $\Lambda_{2 n}$ is introduced. Either at some succeeding stage the symbols $\Lambda_{2 n}$ and $\Lambda_{2 n}^{\prime}$ are both erased (and the function $\phi_{e}$ spoiled) or, since $\phi_{e}$ is total, the marker $\Lambda_{2 n}^{\prime}$ must come to rest permanently beside a number $m$. But this implies that $m$ fails to belong to $K$, and that there exists a number $r$ such that $\phi_{r}(r)=m$, and $r \in L_{0}$, and $\phi_{r}=\phi_{e}$. In view of (iv) of our preliminary discussion, $\phi_{e}$ cannot have the properties described at the beginning of this paragraph. Thus $L_{0}$ is not a 1-1 semicylinder.

Corollary 1 . The sets $L_{0}$ and $L_{1}$ described in the statement of the theorem are bounded-truth-table-complete noncreative sets.

Proof. Since a creative set is necessarily a 1-1 semi-cylinder, it suffices to show that the set $K$ of the theorem is bounded-truth-table reducible to $L_{0}$. Let $f$ be a recursive function such that $x \in L_{1}$ if and only if $f(x) \in L_{0}$. Then $x \in K$ if and only if $x \in L_{0}$ or $f(x) \in L_{0}$, which is an example of bounded-truth-table reducibility.

Corollary 2 (FISCher). Bounded-truth-table reducibility differs from many-one reducibility on the r.e. sets. 
Proof. This is now immediate since all many-one-complete sets are creative.

A noncreative r.e. set $A$ has been called pseudo-creative if for every r.e. set $B \subset A^{\prime}$ there is an infinite r.e. set $C \subset A^{\prime}$ such that $B \cap C=\emptyset$. A standard method for showing that the class of pseudo-creative sets is nonempty is to consider the cylinder of any nonrecursive, noncreative, r.e. set. (See [3].) In another paper [7], we show that there is a pseudo-creative set which is not a cylinder but which is bounded-truth-table reducible to a simple set. Corollary 1 can be used to obtain additional information about pseudo-creative sets which are not cylinders.

Corollary 3. There is a pseudo-creative set which is not a cylinder and which is bounded-truth-table reducible, among all the r.e. sets, only to pseudocreative sets and to creative sets.

Proof. From the following lemma and from the transitivity of boundedtruth-table reducibility, it follows that any bounded-truth-table-complete noncreative set satisfies the conditions of the corollary.

Lemma (Post-Shoenfield). Every bounded-truth-table-complete set is either creative or pseudo-creative.

Proof. In [4] it is proved that no bounded-truth-table-complete set is either recursive or simple. In [6] it is proved that if $A$ is r.e. but not recursive and if there is an infinite r.e. subset $B \subset A^{\prime}$ such that $A \cup B$ is simple (i.e., if $A$ is pseudo-simple), then $A$ is not bounded-truth-tablecomplete. It is easily shown that any r.e. set which is neither recursive, simple, nor pseudo-simple is either creative or pseudo-creative. (See [3].)

REMARK. The proofs in [4] and [6] on which this lemma depends are rather involved. It has been pointed out to the author by T. G. McLaughlin that an easier proof of Corollary 3 is obtained by observing that the class of recursive, simple, and pseudo-simple sets forms a lattice under intersection and union. Hence the sets $L_{0}$ and $L_{1}$ must be either pseudo-creative or creative.

\section{AN INFINITE SPLINTER WHICH IS NOT A CYLINDER}

Introduction. Ullian has defined a splinter to be a set of the form $\left\{f^{i}(n) \mid i \in N\right\}$ where $n$ is some fixed integer and $f$ is a total recursive function. A 1-1 splinter is a splinter of a 1-1 recursive function. It can be shown that an infinite and coinfinite r.e. set is a cylinder if and only if it is an infinite and coinfinite 1-1 splinter. Myhill has raised the question of whether every infinite and coinfinite splinter is a cylinder [3].

In this part of the paper we construct an infinite and coinfinite splinter which is not a cylinder. 
TheOREM. There exists an infinite and coinfinite splinter which is not a semi-cylinder.

Proof. Let $K=\left\{x \mid \phi_{x}(x)\right.$ converges $\}$. Let $g$ be any 1-1 recursive function whose range is $K$.

We will proceed in stages, constructing a total recursive function $h$ such that $S=\left\{h^{i}(1) \mid i \in N\right\}$ will be the desired splinter. $\left\{h^{i}(0) \mid i \in N\right\}$ will be a subset of $S^{\prime}$.

As we proceed with our construction, we will use an infinite collection of sets, $\left\{S_{k}^{(n)}\right\}(k, n \in N)$. For all $k, S_{k}^{(0)}=\emptyset$; if $S_{k}^{(n)} \neq \emptyset, S_{k}^{(n)}$ will be linearly ordered under the relation $a \prec b$ if and only if there is some $i>0$ such that $h^{i}(a)=b$. If $S_{k}^{(n)} \neq \emptyset$, we define $r_{k, 0}^{(n)}$ to be the first member of $S_{k}^{(n)}$ under the order $\prec$, and we define $r_{k, 1}^{(n)}$ to be the last member of $S_{k}^{(n)}$ under the order $\prec$.

We define $o_{n}$ to be that unique $y$ for which there exists $j \geqq 0$ such that $h^{j}(0)=y$ and such that $h(y)$ is not defined by the end of Stage $n$. We define $i_{n}$ to be that unique $z$ for which there exists $j \geqq 0$ such that $h^{j}(1)=z$ and such that $h(z)$ is not defined by the end of Stage $n$.

\section{Construction.}

Stage 0. Define $i_{0}=1$ and $o_{0}=0$.

Stage $n(n>0)$. Let $F_{n}=\left\{y \mid S_{y}^{(n-1)} \neq \emptyset\right.$ and during Stage $n-1$ it was not specified that $\left.S_{y}^{(n)}=\emptyset\right\}$. This is a finite set, so we may suppose it has $p$ members, $y_{1}, y_{2}, \cdots, y_{p}$. Let $l_{0}, l_{1}, \cdots, l_{p}, l_{p+1}$ be the smallest $p+2$ integers which have not appeared in our construction of $h$. Define $h\left(r_{y_{k, 1}}^{(n-1)}\right)=l_{k}$ $(1 \leqq k \leqq p), h\left(o_{n-1}\right)=l_{0}$, and $h\left(i_{n-1}\right)=l_{p+1}$. For $1 \leqq k \leqq p$, define $S_{y_{k}}^{(n)}$ $=S_{y_{k}}^{(n-1)} \cup\left\{l_{k}\right\}$. If $y \notin F_{n}$, we do not yet define $S_{y}^{(n)}$, but instead wait until the end of Stage $n$, at which time we define $S_{y}^{(n)}=\emptyset$ unless $S_{y}^{(n)}$ is defined to be a unit set during Stage $n$, as described below.

Notation. When in the following we say "(re)introduce the marker $\Lambda_{q}$," we mean: find a $z$ such that $z$ has not yet been used as an index, $i$, for some $S_{i}^{(w)}$ with $S_{i}^{(w)} \neq \emptyset$; then find a $q^{\prime}$ such that $q^{\prime}$ has not appeared in our construction of $h$ and such that $\phi_{q^{\prime}}=\phi_{g(q)}$; define $S_{z}^{(n)}=\left\{q^{\prime}\right\}$ and place the marker $\Lambda_{q}$ beside $q^{\prime}$.

Now compute $g(n-1)$ and introduce the marker $\Lambda_{n-1}$, introducing $g(n-1)$ and placing $\Lambda_{n-1}$ beside $g(n-1)$ if $g(n-1)$ has not yet appeared in our construction of $h$. Then if $g(n-1)$ fails to have beside it a marker, $\Lambda_{m}$, go on to Stage $n+1$. Otherwise, as can be seen as the construction is fully described, $g(n-1)=r_{k, 0}^{(n)}$ for some $k$. For the duration of Stage $n$, we abbreviate $r_{k, 0}^{(n)}$ to $r$. Since $g(n-1)=r \in K, \phi_{r}(r)$ converges; we compute $\phi_{r}(r)$. (As the construction is fully described, it will be seen that $\phi_{r}=\phi_{g(m)}$. The remainder of Stage $n$ is an attempt to prevent $\phi_{g(m)}$ from being a function which would make $S$ a semi-cylinder.) 
Figure 1.

The graph of $h$ during the examination of cases in Stage $n$.

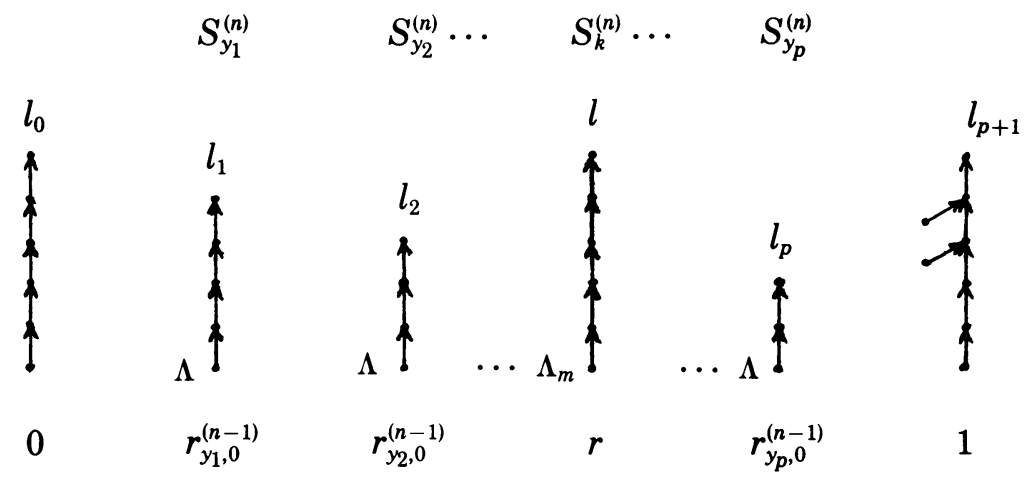

Case A. $\phi_{r}(r)=r$. In this case, $\phi_{g(m)}$ cannot be the function required by the definition if $S$ is to be a semi-cylinder. Erase the marker $\Lambda_{m}$, set $h\left(l_{0}\right)=r$, and define $S_{k}^{(n+1)}=\emptyset$. Then go on to Stage $n+1$.

Case B. $\phi_{r}(r) \in S_{j}^{(n)}$ for $j \neq k$. Let the marker beside $r^{(n)}$ be $\Delta_{x}$.

Subcase I. $x<m$. In this case define $h\left(l_{p+1}\right)=r$. Erase $\Lambda_{m}$, making its removal contingent upon $S_{j}$ remaining in $S^{\prime}$. Now for those markers, $\Lambda_{q_{0}}, \cdots, \Lambda_{q_{d}}$, whose removal was contingent upon $S_{k}$ remaining in $S^{\prime}$, we successively reintroduce the markers $\Lambda_{q_{0}}, \cdots, \Lambda_{q_{d^{*}}}$ We then define $S_{k}^{(n+1)}=\emptyset$ and go on to Stage $n+1$.

Subcase II. $m \leqq x$. (As the construction is fully described, it can be seen that in fact $m$ must be less than $x$.) In this case define $h\left(l_{p+1}\right)=r$ and $h\left(l_{0}\right)$ $=r_{j, 0}^{(n)}$. Erase marker $\Lambda_{x}$ from its position beside $r_{j, 0}^{(n)}$, and then reintroduce the marker $\Lambda_{x}$. For those markers, $\Lambda_{q_{0}}, \cdots, \Lambda_{q_{d}}$, whose removal was contingent upon $S_{k}$ remaining in $S^{\prime}$, successively reintroduce the markers $\Lambda_{q_{0}}, \cdots, \Lambda_{q_{d^{\prime}}}$ Erase marker $\Lambda_{m}$, define $S_{k}^{(n+1)}=\emptyset=S_{j}^{(n+1)}$, and go on to Stage $n+1$.

Case C. $\phi_{r}(r) \in S_{k}^{(n)}$ but $\phi_{r}(r) \neq r$. In this case, define $h\left(l_{p+1}\right)=h(r)$. (This puts $r$ in $S^{\prime}$ and $\phi_{r}(r)$ in $S$.) Erase the marker $\Lambda_{m}$ and set $S_{k}^{(n+1)}=\emptyset$. Now for those markers, $\Lambda_{q_{0}}, \cdots, \Lambda_{q_{d}}$, whose removal was contingent upon $S_{k}$ remaining in $S^{\prime}$, successively reintroduce the markers $\Lambda_{q_{0}}, \cdots, \Lambda_{q_{d^{*}}}$. Then go on to Stage $n+1$. (As the construction is fully described, one can see that for any such $q_{j}, q_{j}>m$.)

Case D. $\phi_{r}(r)=h^{i}(0)$ for some $i \geqq 0$ or $\phi_{r}(r) \in h^{-1} h^{i}(1)$ but $\phi_{r}(r) \neq h^{i-1}(1)$ for some $i>0$. (In this case $\phi_{r}(r) \in S^{\prime}$.) Define $h\left(l_{p+1}\right)=r$, erase marker $\Lambda_{m}$, and define $S_{k}^{(n+1)}=\emptyset$. For those markers, $\Lambda_{q_{0}}, \cdots, \Lambda_{q_{d}}$, whose removal was contingent upon $S_{k}$ remaining in $S^{\prime}$, successively reintroduce the markers $\Lambda_{q_{0}}, \cdots, \Lambda_{q_{d^{*}}}$ Then go on to Stage $n+1$.

Case E. $\phi_{r}(r)=h^{i}(1)$ for some $i \geqq 0$. In this case define $h\left(l_{0}\right)=r$, erase the marker $\Lambda_{m}$, set $S_{k}^{(n+1)}=\emptyset$, and go on to Stage $n+1$. 
Case F. $\phi_{r}(r)$ has not yet appeared in our construction of $h$. In this case, define $h\left(l_{p+1}\right)=\phi_{r}(r)$ and $h\left(l_{0}\right)=r$. Then erase $\Lambda_{m}$, set $S_{k}^{(n+1)}=\emptyset$, and go on to Stage $n+1$.

This completes the description of the construction of $h$.

It is clear that $h$ is a total recursive function and that $S=\left\{h^{i}(1): i \in N\right\}$ is an infinite splinter. In order to show that $S$ is not a semi-cylinder, we first show, by induction, that every marker comes permanently to rest beside some number or is permanently erased.

The marker $\Lambda_{0}$ is introduced at Stage 1 , and can never be moved or reintroduced (though it can be erased).

Now suppose all of the markers $\Lambda_{0}, \cdots, \Lambda_{n-1}$ have been either permanently erased or have come permanently to rest by Stage $m_{0}$. If the marker $\Lambda_{n}$ were not to satisfy these conditions, it would at some Stage $m_{1}$, with $m_{1}>m_{0}$, be beside a number, $r$, from which it would be moved or removed at Stage $m_{1}$. Basically there are only two ways this could occur: either $g\left(m_{1}-1\right)=r$ or $g\left(m_{1}-1\right)=l$ where $l$ has beside it a marker $\Lambda_{q}$ with $q<n$. The latter case cannot occur, for an examination of cases shows that $\Lambda_{q}$ would then be either moved or removed, contradicting our induction hypothesis. In the former case, $\Lambda_{n}$ must be erased and its erasure is permanent unless Subcase I of Case B has obtained. But in this latter event, the removal of $\Lambda_{n}$ is contingent upon $S_{j}$ remaining in $S^{\prime}$, where the first element of $S_{j}^{\left(m_{1}\right)}$ has beside it a marker $\Lambda_{q}$ with $q<n$. Thus reintroducing the marker $\Lambda_{n}$ requires moving or removing the marker $\Lambda_{q}$, contrary to our induction hypothesis.

Now suppose $S$ were a semi-cylinder with a total recursive function $\phi_{e}$ such that $x \in S$ implies $\phi_{e}(x) \in S-\{x\}$ and $x \in S^{\prime}$ implies $\phi_{e}(x) \in S^{\prime}$ $-\{x\}$. Since $\phi_{e}$ is total, $\phi_{e}(e)$ is defined, so $e=g(n)$ for some $n$. At Stage $n+1$ the marker $\Lambda_{n}$ is introduced. If $\Lambda_{n}$ were ever permanently erased, by examining the various cases, we see that $\phi_{e}$ could not be as described. Therefore we may assume that $\Lambda_{n}$ comes permanently to rest beside some number $s$. But by our construction, this implies that $\phi_{e}=\phi_{s}$ and hence, since $\phi_{e}$ is total, $s \in K$. Since $s$ cannot have appeared in our construction before $\Lambda_{n}$ is placed beside it, $s=g\left(n_{0}\right)$ for some $n_{0}>n$, and so at Stage $n_{0}+1$ we compute $\phi_{s}(s)$. Examining our construction, we see that $\Lambda_{n}$ is then either moved or erased. This contradiction shows that $\phi_{e}$ cannot be the desired function, and hence $S$ is not a semi-cylinder. Since both $S$ and $S^{\prime}$ have two elements by the end of Stage 1 , neither is a unit set, and so both must be infinite.

Corollary. There is an infinite and coinfinite splinter which is not a cylinder.

Proof. Every cylinder is a semi-cylinder. 
REMarKs. 1. The set $S$ is "almost" a 1-1 splinter in the following sense: If $x \in S^{\prime}, h^{-1}(x)$ has at most one element. If $x \in S, h^{-1}(x)$ has at most two elements and $h^{-2}(x)$ has at most one element.

2. In Part I and in [7], we have constructed pseudo-creative sets which are not cylinders. Since Ullian has shown that all splinters are recursive, creative, or pseudo-creative (see [3], and see Part I for a definition of pseudo-creative), the set $S$ of the Theorem must also be a pseudo-creative noncylinder. The question of whether all pseudo-creative sets are splinters remains open.

\section{REFERENCES}

1. P. Fischer, A note on bounded-truth-table reducibility, Proc. Amer. Math. Soc. 14 (1963), 875-877.

2. J. Myhill, Creative sets, Z. Math. Logik Grundlagen Math. 1 (1955), 97-108.

3. _ Recursive digraphs, splinters and cylinders, Math. Ann. 138 (1959), 211-218.

4. E. L. Post, Recursively enumerable sets of positive integers and their decision problems, Bull. Amer. Math. Soc. 50 (1944), 284-316.

5. H. Rogers, Jr., Recursive functions and effective computability, McGraw-Hill, New York, (to appear).

6. J. Shoenfield, Quasicreative sets, Proc. Amer. Math. Soc. 8 (1957), 964-967.

7. P. R. Young, A note on pseudo-creative sets and cylinders, Pacific J. Math. 14 (1964), 749-753.

\section{Reed College, Portland, Oregon}

